\title{
Neutrino masses, states and interactions: session summary
}

\author{
Stefano Gariazzo \\ IFIC (CSIC / University of Valencia) \\ E-mail: gariazzodific.uv.es
}

\section{Monica Sisti}

Dipartimento di Fisica, Università di Milano-Bicocca, I-20126 Milano, Italy

INFN-Sezione di Milano-Bicocca, I-20126 Milano, Italy

E-mail: monica.sistiemib.infn.it

In these proceedings we present a brief summary of the parallel Session IV, "Neutrino masses, states and interactions", part of the Neutrino Oscillation Workshop 2018* Topics of the session included the current status of some experimental searches for the neutrino absolute mass scale, a resume of recent results and developments in neutrinoless double beta decay, the situation of the search for a light sterile neutrino with a mass around $1 \mathrm{eV}$, a phenomenological summary on neutrino electromagnetic properties and a discussion about theoretical models for neutrino masses and mixings.

Neutrino Oscillation Workshop (NOW2018)

9 - 16 September, 2018

Rosa Marina (Ostuni, Brindisi, Italy)

${ }^{*}$ All the talks are available on the conference webpage [1]. 


\section{Introduction}

In the context of particle physics, the neutrino sector of the standard model is the less known one. A number of experiments have demonstrated that neutrinos are not massless particles, as the standard model assumes, but at least two of them have a positive mass and therefore can oscillate. Since oscillations only provide us information on the mass splittings, we still ignore the absolute scale of the neutrino masses. Cosmology currently provides a stringent upper limit on the sum of the neutrino masses, much stronger than what has been tested in terrestrial experiments until now. Since cosmological limits depend on the assumptions on the Universe evolution, a measure of neutrino masses at Earth is mandatory to obtain a definitive result.

A very important question concerning neutrinos regards the nature of these particles. Theory allows two possibilities for massive neutral fermions: they can either be of Dirac or of Majorana type. In nature, it seems that only Dirac fermions exist, as no Majorana fermion has been observed yet. A Majorana particle coincides with its antiparticle, thus implying that only neutral particles can be of Majorana type. Neutrinos are the only candidate to become the first Majorana particles, and the smoking gun for this fact would be the observation of the lepton number violating process known as neutrinoless double beta decay. The extreme rarity of this process makes experiments very difficult. Despite this fact, several collaborations are employing different techniques in order to approach the challenge.

On another hand, anomalous results in neutrino oscillation experiments have hinted the possible existence of additional neutrino states. In particular, we have controversial indications in favor of a new neutrino state corresponding to a new mass splitting around $1 \mathrm{eV}^{2}$. Heavier sterile neutrinos are also invoked to explain the smallness of neutrino masses with respect to the masses of the other fermions in the standard model. The search for additional neutrino states (or right handed fermions in general) is therefore a mandatory step towards a complete understanding of the fermion sector of particle physics.

Finally, in order to fully test the standard model, an extensive study of the neutrino interactions is required. Very recently, the coherent elastic neutrino-nucleous scattering has been observed for the first time. This first measure and its future improvements allow us to open a window on a series of new studies concerning various neutrino properties, including neutrino electromagnetic properties such as its charge radius. Electromagnetic properties of neutrinos, however, can also be constrained through astrophysics, for example one can constrain the neutrino magnetic moment by considering ultra-high energy neutrinos which traverse the interstellar magnetic field.

Parallel Session IV of the Neutrino Oscillation Workshop (NOW) 2018 was devoted to discuss all these themes, as briefly reported here.

\section{Neutrino masses}

The direct measurement of the neutrino mass in laboratory experiments can be pursued by two complementary approaches: the precise study of the shape of a beta spectrum at its kinematical end-point and the search for neutrinoless double beta decay. Though the sensitivity on the effective neutrino mass expected for neutrinoless double beta decay is higher, this nuclear process requires the neutrino to be a Majorana particle. On the other hand, the study of the kinematics of the 
electrons emitted in a beta decay is the most sensitive model independent method to assess the absolute neutrino mass scale.

Current best upper limit on the electron antineutrino mass $\left(m_{v}<2.05 \mathrm{eV}\right.$ at $\left.95 \% \mathrm{CL}\right)$ had been set by the Mainz [2] and Troitsk [3] experiments several years ago. KATRIN, the last generation high resolution spectrometer, will use a high luminosity gaseous tritium source to study with unprecedented precision the spectral distortions close to the end point of the $\beta$-decay of tritium by means of a $10 \mathrm{~m}$ diameter MAC-E filter (Magnetic Adiabatic Collimation with Electrostatic filter). Electrons emitted by the source are selected in energy by a Pre-Spectrometer (also a MAC-E filter of $1.7 \mathrm{~m}$ diameter) so that only the most energetic ones, carrying the information about the antineutrino mass, can enter the Main-Spectrometer. Here their kinetic energy is measured very precisely before they are guided to the counting detector, a Si-PIN diode wafer.

KATRIN started its commissioning phase on May 2018. As V. Sibille explained [4], these preliminary operations demonstrated the high stability of the tritium atom injection as well as the stability of the counting rate on the detector. In the meantime, the theoretical description of the tritium $\beta$-decay spectrum close to the end-point has been carefully studied together with the spectrometer response function. The inclusion of the systematic uncertainties in the model framework is in progress and will be finalized when the commissioning and characterization of the experimental apparatus will be completed. The first neutrino mass runs are expected in early 2019.

A completely complementary approach to the direct neutrino mass measurement is offered by calorimetric experiments. In an ideal calorimeter, the source is embedded in the detector so that only the neutrino energy escapes detection, and all the effects related to decays on excited states, source self-absorption or backscattering - typical of spectrometers - are in principle negligible. On the other hand, a calorimeter measures the whole beta spectrum at once and, given the intrinsic slowness of the detector technology, this poses a serious limitation on the source strength - to avoid spectral distortions due to event pile-up - and on the statistics that can be acquired. For this reason, calorimetric experiments are made of large arrays of high energy resolution single pixels with optimized concentration of the $\beta$-decaying isotope. The technology of low temperature detectors has made the calorimetric approach to the measurement of the neutrino mass feasible [5].

The HOLMES experiment [6] will study the electron capture decay of ${ }^{163} \mathrm{Ho}$ (end-point energy $2.8 \mathrm{keV}$ ) for a sensitive assessment of the electron neutrino mass - as suggested by A. De Rujula and M. Lusignoli in 1982. The adopted technology envisages low temperature detectors made of transition edge sensors coupled to Au absorbers with implanted ${ }^{163} \mathrm{Ho}$ nuclei, for a single pixel activity of $\sim 300 \mathrm{~Hz}$. The goal is to reach a final detector configuration of 1000 channels, each with $1 \mathrm{eV}$ FWHM energy resolution and $1 \mu$ s time resolution. E. Ferri [7] presented the status of the holmium source production, of the ion implanter construction, of the detector array fabrication, and of the microwave read-out multiplexing characterization. A first high statistics ${ }^{163} \mathrm{Ho}$ run with a 64-channel demonstrator is foreseen in 2019.

\section{Neutrinoless double beta decay}

Neutrinoless double beta decay $(0 v \beta \beta)$ is an extremely rare nuclear transition speculated long time ago but never observed. It is expected for a number of even-even nuclei whose single beta decay is energetically forbidden. Its discovery would be a major step in particle physics since it 
would prove lepton number violation and demonstrate the Majorana nature of neutrinos, besides giving important information concerning the neutrino mass scale. As a consequence, many experimental programs are nowadays facing the effort of looking for this decay with different techniques. The goal is to search for the two electron signal - a peak in the summed electron energy spectrum at the Q-value of the transition - with the highest possible sensitivity. To this aim, the background of spurious counts in the energy region of interest must be kept as low as possible, ideally below the intrinsic limit set by the allowed two-neutrino double beta decay continuous spectrum, while the mass of the $0 v \beta \beta$ candidate isotope should be scalable to the tonne scale or larger. A good detector energy resolution is important too, not only to avoid the broadening of the upper tail of the two-neutrino spectrum in the region of interest, but also to single out the $0 v \beta \beta$ peak over an almost flat background.

Recent results on a selection of the current experimental programs were presented at this session.

KamLAND-Zen is an experiment looking for the $0 v \beta \beta$ decay of ${ }^{136} \mathrm{Xe}$ by means of a liquid scintillation detector loaded with isotopically enriched Xe gas. The Xe-loaded scintillator is contained in an inner nylon balloon at the center of the 1 kton liquid scintillator of the KamLAND detector in the Kamioka mine (Japan). The outer scintillator serves as an active veto. The scintillation light is read by 1879 photomultiplier tubes. The energy resolution is $\sigma \sim 7 \% / \sqrt{E(\mathrm{MeV})}$ and the ${ }^{136} \mathrm{Xe}$ Q-value is $2458 \mathrm{keV}$. The first phase of KamLAND-Zen 400 experiment started in 2011 with $320 \mathrm{~kg}$ of Xe and was upgraded in 2013 to $383 \mathrm{~kg}$ of Xe, after a major purification of the Xe-loaded scintillator to remove a ${ }^{110 m} \mathrm{Ag}$ contamination that limited the Phase-I sensitivity. The final result of KamLAND-Zen 400 Phase-I and Phase-II combined analysis is a lower limit on the $0 v \beta \beta$ half-life of ${ }^{136} \mathrm{Xe}$ equal to $T_{1 / 2}>1.07 \times 10^{26} \mathrm{y}$ at $90 \% \mathrm{C}$.L., corresponding to an upper limit on the effective Majorana mass of $m_{\beta \beta}<61-165 \mathrm{meV}$ (depending on the values of the nuclear matrix elements). Y. Gando [8] then presented KamLAND-Zen 800, that will use $750 \mathrm{~kg}$ of Xe in a slightly bigger inner balloon. Great care was put in the production of the nylon balloon in ultra low background conditions, and in the purification of the liquid scintillator. The new inner balloon was installed in May 2018. After the Xe loading, the physics run are foreseen before the end of 2018.

GERDA is searching for the $0 v \beta \beta$ decay of ${ }^{76} \mathrm{Ge}$ by means of enriched high purity Ge (HPGe) detectors with an excellent FWHM energy resolution, $\sim 0.1 \%$ at the ${ }^{76} \mathrm{Ge}$ Q-value of $2039 \mathrm{keV}$. The experimental set-up is located in the Gran Sasso Underground Laboratory (Italy) and for GERDA Phase-II it was upgraded with $35.6 \mathrm{~kg}$ of enriched Ge. As Ch. Wiesinger explained [9], the bare detectors are operated in a radiopure cryogenic liquid (LAr) that cools the detectors at their operating temperature and shields them from the external background. The LAr cryogenic tank is then surrounded by $590 \mathrm{~m}^{3}$ of ultra-pure water that completes the passive shielding. A curtain of wavelength shifting fibers read at both ends by silicon photomultipliers surrounds the HPGe detectors to veto those background events depositing energy in the LAr itself. Additionally, a set of 16 low background photomultipliers suitable for cryogenic use complements the LAr veto system. GERDA Phase-II started the data taking in December 2015 and, with an excellent duty cycle of $92.9 \%$, took data until April 2018. The acquired data, combined to those of Phase-I, sum up to $82.4 \mathrm{~kg} \cdot \mathrm{y}$ (total mass), by far the largest exposure ever achieved for a ${ }^{76} \mathrm{Ge}$ experiment. Thanks to the LAr veto and to the active background suppression through pulse shape discrimination, the remarkable background index of less than $6 \times 10^{-4}$ counts $/(\mathrm{keV} \cdot \mathrm{kg} \cdot \mathrm{y})$ around the Qvalue has been achieved. This translates in a lower limit on the $0 v \beta \beta$ half-life of ${ }^{76} \mathrm{Ge}$ equal to 
$T_{1 / 2}>0.9 \times 10^{26}$ y at $90 \%$ C.L., corresponding to an upper limit on the effective Majorana mass of $m_{\beta \beta}<111-260 \mathrm{meV}$ (depending on the values of the nuclear matrix elements). Upgrades to the LEGEND-200 experiment, with a sensitivity goal of $10^{27} \mathrm{y}$ on the ${ }^{76} \mathrm{Ge}$ half-life, are in preparation.

CUORE is the largest cryogenic $0 v \beta \beta$ decay experiment ever built. It is located in the Gran Sasso Underground Laboratory (Italy) and it is made of $988 \mathrm{TeO}_{2}$ thermal detectors - for a total mass of $742 \mathrm{~kg}$ - containing natural ${ }^{130} \mathrm{Te}$ as $0 v \beta \beta$ candidate isotope. The array is arranged in 19 towers of 52 detectors each and is enclosed in a custom made cryogen-free cryostat that cools them to the operating temperature of $11 \mathrm{mK}$. The cryogenic apparatus hosts also internal lead shields all around the detector array (the bottom and lateral lead shields are made of ancient Roman lead, free from the ${ }^{210} \mathrm{~Pb}$ isotope). Together with the low radioactivity copper of the cryostat thermal shields, the total mass kept at temperatures below $4 \mathrm{~K}$ is of about 15 tons. The system is carefully designed to reduce mechanical vibrations on the detectors and is surrounded by a passive shielding made of $\sim 25 \mathrm{~cm}$ of lead and $\sim 20 \mathrm{~cm}$ of neutron shield. All materials employed to construct the cryogenic apparatus, in particular those in close vicinity to the detectors, have been carefully selected for their radiopurity and the assembly of the detector towers has been made in a clean room environment. As P. Gorla discussed [10], CUORE data taking started in 2017, proving the good performance of the detector and the effectiveness of the background reduction strategy. At the ${ }^{130} \mathrm{Te} \mathrm{Q}$-value $(2528 \mathrm{keV})$, the FWHM effective energy resolution is of $7.7 \mathrm{keV}$ and the background level amounts to 0.014 counts $/(\mathrm{keV} \cdot \mathrm{kg} \cdot \mathrm{y})$ after a $\mathrm{TeO}_{2}$ exposure of $86.3 \mathrm{~kg} \cdot \mathrm{y}$. Combined with precursor $\mathrm{TeO}_{2}$ experiments (Cuoricino and CUORE-0), the achieved lower limit on the $0 v \beta \beta$ half-life of ${ }^{130} \mathrm{Te}$ equals $T_{1 / 2}>1.5 \times 10^{25}$ y at $90 \%$ C.L., corresponding to an upper limit on the effective Majorana mass of $m_{\beta \beta}<110-520 \mathrm{meV}$ (depending on the values of the nuclear matrix elements). More results are expected in the future as the experiment keeps on collecting data.

CUPID-0 represents the latest advancement of the thermal detector technology in terms of background reduction. The detector is an array of $26 \mathrm{ZnSe}$ scintillating thermal detectors with double read-out (heat and light) to search for the $0 v \beta \beta$ decay of ${ }^{82} \mathrm{Se}$ (Q-value equal to $2998 \mathrm{keV}$ ). Exploiting the differences in the light yield between alpha and beta/gamma particles depositing energy in the detector, a powerful particle identification is possible by comparing the heat signal and the light one after each interaction, thus rejecting the dangerous alpha background that is one of the major components of the spurious counts in the region of interest of the CUORE experiment. As N. Casali discussed [11], 24 out of the $26 \mathrm{ZnSe}$ crystals are enriched in ${ }^{82} \mathrm{Se}$, for a total mass of $9.6 \mathrm{~kg}$ for the $0 v \beta \beta$ decay candidate isotope. CUPID- 0 experiment is located in the Gran Sasso Underground Laboratory (Italy) in the same facility that hosted the Cuoricino and CUORE-0 experiments. It started taking data in 2017 and collected a total of $5.74 \mathrm{~kg} \cdot \mathrm{y} \mathrm{ZnSe}$ exposure. At the Q-value, the FWHM resolution of the detectors is of $23 \mathrm{keV}$, while the achieved background index is of $3.2 \times 10^{-3} \mathrm{counts} /(\mathrm{keV} \cdot \mathrm{kg} \cdot \mathrm{y})$. This translates in a lower limit on the $0 v \beta \beta$ half-life of ${ }^{82} \mathrm{Se}$ equal to $T_{1 / 2}>4.0 \times 10^{24} \mathrm{y}$ at $90 \%$ C.L. Since the experiment is currently taking data, more results are expected in the near future.

\section{Light sterile neutrino}

The proposal of the existence of additional neutrino states is not new. Sterile neutrinos, i.e. singlets of the standard model, would only interact with the known matter through gravitational 
interaction and oscillations with the active neutrinos. Depending on their mass, they may be considered as possible candidates for dark matter or play a significant role in the determination of the masses of the light neutrinos, through the Seesaw mechanism.

In the recent years, sterile neutrinos with a mass around $1 \mathrm{eV}$ have been studied as a possible solution for the Short-BaseLine (SBL) neutrino oscillation anomalies, which include results from LSND [12] and MiniBooNE [13], from GALLEX and SAGE [14] and from a number of reactor antineutrino experiments [15]. These experimental measurements cannot be explained in the context of standard three neutrino oscillations. Y.F. Li [16] reviewed the current status of the search of such light sterile neutrino using all the available appearance and disappearance data in SBL experiments. In his talk, he showed how muon (anti)neutrino disappearance as constrained mainly by the IceCube and MINOS+ experiments is substantially in tension with the observation of appearance of electron (anti)neutrinos in a flux of muon (anti)neutrinos as observed by LSND and MiniBooNE, when also the electron (anti)neutrino disappearance results are considered [17]. From this latter channel, however, we have the first model-independent indications [18] in favor of active-sterile neutrino oscillations, thanks to the observations from the NEOS [19] and DANSS [20] experiments. The two collaborations aim at measuring the reactor antineutrino flux at several different distances in order to distinguish the effect related to a global normalization, which does not depend on the distance at which the measurement is performed, from the one due to neutrino oscillations, which instead varies with the baseline.

One of the two experiments, DANSS [20], was discussed in details in the dedicated talk by D. Svirida [21]. DANSS is located below the reactor core of the Kalinin nuclear power plant, in Russia, and uses a movable detector of plastic scintillator in order to detect electron antineutrinos using the Inverse Beta-Decay (IBD) reaction. The detector uses a lifting platform that allows to change its distance from the reactor core in the range between $\sim 10.7 \mathrm{~m}$ (top) and $\sim 12.7 \mathrm{~m}$ (bottom), with an intermediate position at $\sim 11.7 \mathrm{~m}$. Using the bottom/top ratio of the observed spectra with a total of 966k events [20], DANSS obtains a preference for active-sterile oscillations with a $\Delta \chi^{2} \simeq-13$ with respect to the three-neutrinos only scenario, corresponding to a $\sim 2.8 \sigma$ significance. The preliminary results of the analysis of the middle/top ratio confirm the preference, though with a smaller significance. The detector entered the second data taking in May 2018, so more data are expected in the following months.

NEOS and DANSS are not the only two ongoing experiments which use the reactor antineutrinos to measure SBL oscillations. Discussed by L. Kalousis [22], the SoLiD experiment at the BR2 research reactor (Belgium) is looking for neutrino oscillations at distances between 6 and $9 \mathrm{~m}$. The detector of the Phase I is made of 5 modules of 10 planes each, where each plane is a grid of $16 \times 16$ cubes of non-flammable scintillator, for a total of 1.6 tons of active mass. The high granularity helps to efficiently reject the background, in particular to distinguish IBD events from fast neutrons. After an initial commissioning phase, the detector is now running in physics mode since February 2018. The first results will be presented after the calibration and validation of the results will be completed. The structure of the detector will in principle allow to reconstruct the oscillation pattern as a function of both the energy and the distance in a very precise way, while the purity of the $B R 2$ reactor will be useful to study the antineutrino spectrum from ${ }^{235} \mathrm{U}$, with a particular focus on the origin of the spectral distortion around $5 \mathrm{MeV}$, usually called "bump" or "shoulder", which has been observed by several other experiments in the recent years. 


\section{Neutrino electromagnetic properties}

Two of the talks in the session were devoted to the discussion of neutrino electromagnetic (EM) properties such as the charge radius and the magnetic moment.

C. Giunti [23] discussed how to use measurements of coherent elastic neutrino-nucleous scattering (CEvNS) to study the charge radius of the neutrinos. The CEvNS cross section depends both on the neutron and proton form factors, but the dominant contribution is the one coming from neutrons. Using the COHERENT measurement of CEvNS, it is possible to derive bounds on the neutron distribution inside a nucleous, which before 2017 was only probed by neutral-current weak interactions through parity-violating electron scattering. Although the current results derived from COHERENT data have a larger uncertainty with respect to the previous determinations, future experiments will allow a better determination of the neutron distribution inside nuclei. At the same time, it is possible to put bounds on the neutrino charge radius, which is a consequence of radiative corrections which generate neutrino-photon interactions at 1-loop level (see e.g. Ref. [24]). Again, current COHERENT results do not allow to put competitive bounds on the neutrino charge radii, but upcoming experiments will allow a significant improvement of the current precision, so that interesting results should be expected.

In another talk, neutrino magnetic properties, and in particular the neutrino magnetic moment, were more in general discussed by A. Studenikin [25]. Our current knowledge of neutrinos is compatible with null EM properties, but a number of theoretical and experimental studies are devoted to further improve the current bounds and the present understanding of such properties. In particular, from the theory point of view we know that neutrinos can have a magnetic moment only if they are not massless. The exact calculation depends on the mechanism that generates the neutrino masses, but in all the theories the neutrino magnetic moment is proportional to the neutrino mass and several orders of magnitude smaller than the Bohr magneton. The presence of a magnetic moment could also generate new neutrino oscillations for ultra-high energy neutrinos travelling from far astrophysical sources in the interstellar magnetic field. A review of the different probes which are sensitive to the neutrino magnetic moment has been performed, and future perspectives have been briefly discussed at the end of the talk.

\section{Neutrino models}

Neutrino models have been discussed in two talks. The first one, from A. Romanino [26], deals with flavor symmetries in a generic sense. The main questions rotate around the possibility to describe the known neutrino masses in an approximate way without the need of invoking symmetry breaking. Another interesting question involves the possible connection between studying flavor symmetries at high and at low scales, in the symmetric limit. The answer to the first question is that, if a normal hierarchy for the neutrino masses will be confirmed by oscillation experiments [27], symmetry breaking will be required in order to explain the mass patterns in the lepton sector, as general symmetries can only produce inverted hierarchical or anarchical mass patterns. Concerning the second question, it can be shown that the low-scale version of a representation is equivalent to the high-scale only if the symmetry satisfies specific conditions. If this does not happen, the 
non-equivalence between low- and high-scale representations could allow to reproduce normal hierarchical neutrino masses even without invoking symmetry breaking.

The second talk, by S. Zajac [28], was devoted to study lepton masses in the context of two Higgs doublet models (2HDM). The motivation comes from the fact that it is difficult to generate Yukawa couplings using discrete symmetries in the context of single Higgs doublet models. After introducing a discrete flavor symmetry in the $2 \mathrm{HDM}$ scenario, one can perform the calculation of the non-trivial mass matrix in the Dirac case, but the result is that it is impossible to reproduce the known lepton mixing matrix, neutrino and charged lepton masses. In the Majorana case the solution of the problem is more complicated, but the conclusions are the same: no discrete symmetry can generate the observed mass spectrum for the charged leptons and the known neutrino mixing matrix. The conclusion is therefore valid independently of the nature of neutrino masses and models with more than two Higgs doublets may be required.

\section{Acknowledgements}

We would like to thank the organizers for inviting us to chair this parallel session.

SG receives support from the European Union's Horizon 2020 research and innovation programme under the Marie Skłodowska-Curie individual grant agreement No. 796941, and by the Spanish grants FPA2015-68783-REDT, FPA2017-90566-REDC (Red Consolider MultiDark), FPA201785216-P, SEV-2014-0398 (AEI/FEDER, UE, MINECO), PROMETEOII/2018/165 and GV2016142 (Generalitat Valenciana).

\section{References}

[1] http://www.ba.infn.it/ now/now2018/program.html.

[2] C. Kraus, B. Bornschein, L. Bornschein, J. Bonn, B. Flatt, A. Kovalik et al., Final results from phase II of the Mainz neutrino mass searchin tritium $\beta$ decay, The European Physical Journal C - Particles and Fields 40 (2005) 447.

[3] V. N. Aseev, A. I. Belesev, A. I. Berlev, E. V. Geraskin, A. A. Golubev, N. A. Likhovid et al., Upper limit on the electron antineutrino mass from the Troitsk experiment, Phys. Rev. D 84 (2011) 112003.

[4] V. Sibille, "First tritium results of the KATRIN analysis." Talk given at NOW 2018, these proceedings, 2018.

[5] A. Nucciotti, The Use of Low Temperature Detectors for Direct Measurements of the Mass of the Electron Neutrino, Advances in High Energy Physics 2016 (2016) 9153024.

[6] B. Alpert, M. Balata, D. Bennett, M. Biasotti, C. Boragno, C. Brofferio et al., Holmes, The European Physical Journal C 75 (2015) 112.

[7] E. Ferri, "The HOLMES experiment: status and perspectives." Talk given at NOW 2018, these proceedings, 2018.

[8] Y. Gando, "Neutrinoless double beta decay search with KamLAND-Zen.” Talk given at NOW 2018, these proceedings, 2018.

[9] C. Wiesinger, "Recent results from GERDA Phase II." Talk given at NOW 2018, these proceedings, 2018. 
[10] P. Gorla, "Latest results from the CUORE experiment." Talk given at NOW 2018, these proceedings, 2018.

[11] N. Casali, "CUPID-0:cryogenic calorimeters with light and heat read-out for $0 v \beta \beta$ searches ." Talk given at NOW 2018, these proceedings, 2018.

[12] LSND collaboration, A. Aguilar-Arevalo et al., Evidence for neutrino oscillations from the observation of anti-neutrino(electron) appearance in a anti-neutrino(muon) beam, Phys.Rev.D 64 (2001) 112007 [hep-ex/0104049].

[13] MiniBooNE collaboration, A. Aguilar-Arevalo et al., Observation of a Significant Excess of Electron-Like Events in the MiniBooNE Short-Baseline Neutrino Experiment, 1805.12028.

[14] C. Giunti and M. Laveder, Statistical Significance of the Gallium Anomaly, Phys.Rev.C 83 (2011) $065504[1006.3244]$.

[15] G. Mention, M. Fechner, T. Lasserre, T. A. Mueller, D. Lhuillier, M. Cribier et al., The Reactor Antineutrino Anomaly, Phys.Rev.D 83 (2011) 073006 [1101.2755].

[16] Y. Li, "Status of eV-scale sterile neutrinos ." Talk given at NOW 2018, these proceedings, 2018.

[17] S. Gariazzo, C. Giunti, M. Laveder and Y. F. Li, Updated Global 3+1 Analysis of Short-BaseLine Neutrino Oscillations, JHEP 06 (2017) 135 [1703.00860].

[18] S. Gariazzo, C. Giunti, M. Laveder and Y. F. Li, Model-Independent $\bar{v}_{e}$ Short-Baseline Oscillations from Reactor Spectral Ratios, Phys.Lett. B782 (2018) 13 [1801.06467].

[19] Y. J. Ko et al., Sterile Neutrino Search at the NEOS Experiment, Phys.Rev.Lett. 118 (2017) 121802 [1610.05134].

[20] DANSS collaboration, I. Alekseev et al., Search for sterile neutrinos at the DANSS experiment, Phys.Lett. B787 (2018) 56 [1804.04046].

[21] D. Svirida, "Searches for sterile neutrinos at the DANSS experiment." Talk given at NOW 2018, these proceedings, 2018.

[22] L. Kalousis, "SoLid: Search for oscillations with a Li-6 detector." Talk given at NOW 2018, these proceedings, 2018.

[23] C. Giunti, "Neutrino EM properties from coherent elastic -nucleus scattering." Talk given at NOW 2018, these proceedings, 2018.

[24] C. Giunti and A. Studenikin, Neutrino electromagnetic interactions: a window to new physics, Rev.Mod.Phys. 87 (2015) 531 [1403.6344].

[25] A. Studenikin, "Magnetic moment of the neutrino." Talk given at NOW 2018, these proceedings, 2018.

[26] A. Romanino, "Unbroken flavour symmetry vs lepton masses and mixings." Talk given at NOW 2018, these proceedings, 2018.

[27] P. De Salas, S. Gariazzo, O. Mena, C. Ternes and M. Tórtola, Neutrino Mass Ordering in 2018: Global Status, 1806.11051.

[28] S. Zajac, "Lepton masses and mixing in Two-Higgs-doublet model." Talk given at NOW 2018, these proceedings, 2018. 